\begin{tabular}{|c|c|}
\hline 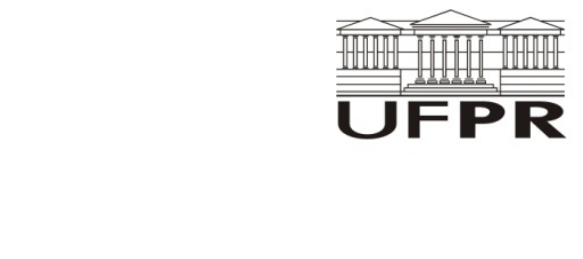 & $\begin{array}{r}\text { SISTEMA } \\
\text { ELETRÔNICO } \\
\text { DE REVISTAS } \\
\text { SER | UFPR } \\
\\
\text { www.ser.ufpr.br }\end{array}$ \\
\hline
\end{tabular}

\title{
Agroecología situada: contexto, proceso y masificación del Programa de Formación en Agroecología en Venezuela ${ }^{1}$
}

\author{
Agroecologia situada: contexto, processo e massificação do \\ Programa de Capacitação em Agroecologia na Venezuela
}

\section{Situated agroecology: context, process and massification of Program Degree in Agroecology in Venezuela}

\author{
Olga DOMENÉ-PAINENAO ${ }^{1}$, Francisco F. HERRERA ${ }^{2}$ \\ ${ }^{1}$ El Colegio de la Frontera Sur (ECOSUR), San Cristóbal de Las Casas, Chiapas, México. \\ ${ }^{2}$ Instituto Venezolano de Investigaciones Científicas (IVIC), Caracas, DF, Venezuela. \\ *E-mail de contacto: oedomene@ecosur.edu.mx
}

Artículo recibido en 4 de junio de 2021, publicado en 30 de noviembre de 2021.

RESUMEN: A lo largo del proceso de la Revolución Bolivariana, se han desarrollado en Venezuela fuertes contradicciones de clase y del sistema alimentario, que han enfrentado las fuerzas de una cultura de consumo que favorece los alimentos importados, el modelo de la revolución verde, y el uso intensivo de insumos, a otro modelo que representa la ruta hacia la soberanía alimentaria y un paradigma agroecológico emergente impulsado por los movimientos de base. Como producto del proceso revolucionario, en 2004 se fundó en la Universidad Bolivariana de Venezuela, el Programa de Formación de Grado en Agroecología (PFG) con el fin de ampliar las prácticas y conocimientos agroecológicos con base en enfoques pedagógicos alternativos. En la pasada década, más de mil graduados del PFG han ocupado espacios institucionales y proyectos productivos en áreas urbanas y rurales, contribuyendo así a la escala agroecológica vertical y horizontal, lo que muestra su papel clave en este creciente movimiento y su capacidad de crear mediadores para territorializar la agroecología e institucionalizar la política pro campesina en Venezuela. Como dato político atípico, este país es un caso

\footnotetext{
Este artículo fue publicado originalmente en inglés en: Domené-Painenao, O.; Herrera, F. F. Situated agroecology: massification and reclaiming university programs in Venezuela. Agroecology and Sustainable Food Systems, 43(7-8), 936-953, 2019.
} 
importante para el estudio de las estrategias de territorialización de la agroecología socialmente comprometida y situada.

Palabras claves: agroecología; soberanía alimentaria; Revolución Bolivariana; educación; políticas públicas.

RESUMO: Ao longo do processo de transformação conhecido como Revolução Bolivariana desde 1999, duras contradições de classe se desenvolveram na Venezuela e com o sistema alimentar, que opôs as forças de uma cultura de consumo que favorece os alimentos importados, o modelo agrário da Revolução Verde, uso intensivo de insumos, em comparação com outro modelo que representa um impulso do Estado em direção à soberania alimentar, e um paradigma agroecológico emergente impulsionado por movimentos populares, que conquistaram aberturas políticas por meio um ambiente político amplamente favorável. Na Universidade Bolivariana da Venezuela, um produto do processo revolucionário, o Programa de Graduação em Agroecologia (PFG) foi fundado em 2004 para expandir as práticas e conhecimentos agroecológicos, com base em abordagens pedagógicas alternativas. Na última década, mais de mil egressos do PFG passaram a ocupar espaços institucionais e projetos produtivos em áreas urbanas e rurais, contribuindo para a escala agroecológica vertical e horizontal. Os formados e educadores do PFG desempenham um papel fundamental no crescente movimento da agricultura urbana que enfrenta a crise econômica. O PFG criou mediadores chave na forma de talento humano para territorializar a agroecologia e institucionalizar a política pró-camponesa na Venezuela. Como dado político atípico, a Venezuela é um caso importante para o estudo das estratégias de territorialização do que chamamos de agroecologia socialmente comprometida e situada.

Palavras-chave: agroecologia; soberania alimentar; Revolución Bolivariana; Educação; políticas públicas.

ABSTRACT: Throughout the process of transformation since 1999 known as the Bolivarian Revolution, harsh class and food system contradictions have unfolded in Venezuela, pitting against one another the forces of the pervasive consumer culture favoring imported foods, the input-intensive Green Revolution agricultural model that represents a state-led push toward food self-sufficiency, and an emerging agroecological paradigm pushed forward by grassroots movements who have seized political openings through the largely supportive policy environment. The Bolivarian University of Venezuela, itself a product of the revolutionary process, founded the Program Degree in Agroecology in 2004 to expand agroecological practices and knowledge, based on alternative pedagogical approaches. Over the next decade, over a thousand PFG graduates have come to occupy institutional spaces and productive projects in urban and rural areas, contributing to vertical and horizontal agroecological scaling. PDA graduates and educators play a key role in the growing movement of urban agriculture that confronts the economic crisis. The PDA has created key mediators in the form of human talent for territorializing agroecology and institutionalizing pro-peasant policy in Venezuela. As a political outlier, Venezuela is an important case for studying the strategies for territorializing what we refer to as socially committed, situated agroecology.

Keywords: agroecology; food sovereignty; Revolución Bolivariana; education; public policies.

\section{Introducción}

La dependencia del petróleo ha caracterizado la vida cultural, política y económica de Venezuela desde principios del siglo XX, dando lugar a presiones externas (Domené-Painenao et al.,
2015; Schiavoni, 2015; Herrera et al., 2017; Felicien et al., 2018a). A finales de la década de 1920, el predominio del petróleo en la economía nacional de este país generó un fuerte efecto del síndrome holandés (Corden, 1984), fortaleciendo la moneda, fomentando la importación de 
productos alimenticios, y propiciando que un país subdesarrollado se incorporase a los países en vías de desarrollo. La agricultura nacional no era rentable para extranjeros y locales, de modo que una elevada tasa de migración de las zonas rurales a las ciudades provocó una gran expansión de la pobreza urbana. Esta situación colocó a Venezuela como una nación dependiente del petróleo, y esto determinó que su sector agrícola y soberanía alimentaria fueran débiles durante la segunda mitad del siglo XX (Espinoza, 2009). A finales de los años ochenta, la mayoría de la población sufre un grave deterioro en su calidad de vida debido al ajuste estructural de las políticas liberales establecidas por el sistema financiero mundial, lo cual condujo a los disturbios antineoliberales del Caracazo de 1989 (Contreras, 2004; Verger \& Muhr, 2007). La elección de Hugo Chávez como presidente en 1998 marcó el inicio del intento de pasar de una cultura rentista dependiente del petróleo y de los suministros agrícolas extranjeros a un modelo diferente que por entonces aún no estaba definido, pero que se oponía claramente al ajuste estructural. El proceso a cargo de la Asamblea Constituyente de 1999, que culminó con una Carta Magna aprobada por el $72 \%$ de los votantes, abrió un profundo debate sobre los problemas de la vida nacional e identificó la soberanía alimentaria como un derecho fundamental que el Estado tiene la responsabilidad de defender.

Fue necesaria la transformación de muchas instituciones públicas y la creación de otras para empezar a garantizar los derechos que habían sido definidos por la Constitución de 1999. El acceso a los alimentos, la atención de la salud, la educación, la vivienda y el desarrollo industrial requieren inversión pública rápida y sostenida. Se identificó entonces que hacía falta un sistema de educación universitaria menos elitista, con el fin de proporcionar los recursos humanos que permitieran satisfacer las demandas emergentes. La Universidad Bolivariana de Venezuela (UBV) se fundó en 2003 como un medio para desafiar la estructura convencional y colonial del sistema universitario (Chiroleu, 2009; D’Amario, 2009). La UBV se fundó no solo con la idea de extender la educación convencional a una mayor parte de la población, sino también de descubrir y recuperar nuevos tipos de conocimiento para responder a las demandas de una sociedad en proceso de descolonización (Giraldo \& McCune, 2019).

Para responder a la necesidad de incluir el conocimiento indígena y campesino en la producción de alimentos, la UBV ofreció el Programa de Formación de Grado en Agroecología (PFG), una carrera de pregrado basada en el objetivo explícito de ampliar esa rama del conocimiento. El PFG se diseñó sobre los pilares de la inclusión social, la pertinencia territorial y la organización comunitaria (Documento Rector, 2003; Domené, 2013), a partir de una concepción de investigación acción participativa de la agroecología (Méndez et al., 2013).

Este texto examina los conceptos, trayectoria e impacto del PFG en Venezuela durante el periodo 2004-2016, utilizando el marco de escalamiento agroecológico definido por Mier y Terán et al. (2018), así como la amplia experiencia como participantes-observadores situados que los autores han tenido en este proceso. Utilizamos entrevistas con profesores y estudiantes para examinar la relación entre el PFG y los principales impulsores de la agroecología en el contexto único y altamente politizado del intento de dos décadas de Venezuela 
de abrirse camino fuera del capitalismo y los diversos métodos que las élites venezolanas y los imperialistas estadounidenses han utilizado para prevenir los éxitos, limitar la influencia y revertir el legado de la Revolución Bolivariana.

\section{Masificación agroecológica: el contexto venezolano}

Varios autores han contribuido a la comprensión teórica de los procesos de masificación agroecológica (Parmentier, 2014; Rosset, 2015b; Rosset \& Altieri, 2017; Mier y Terán et al., 2018). Se ha sugerido un conjunto de impulsores principales basados en casos seleccionados en los que las organizaciones y movimientos rurales representan a sujetos transformados y cuyo objetivo final es la expansión geográfica. Estos impulsores de la ampliación, definidos por Mier y Terán et al. (2018), pueden actuar solos o asociados, e incluyen: 1) crisis que impulsan la búsqueda de alternativas; 2) organización social; 3) procesos constructivistas de enseñanzaaprendizaje; 4) prácticas agroecológicas efectivas; 5) movilización del discurso; 6) aliados externos; 7) mercados favorables, y 8) oportunidades políticas y políticas favorables. Sin embargo, Parmentier (2014) sugiere un conjunto de recomendaciones para la masificación agroecológica a través de una estrategia vertical para alcanzar una etapa avanzada. Las recomendaciones se basan sobre todo en políticas institucionales para ayudar a desbloquear las barreras ideológicas; apoyar a las redes de agricultores a agricultores; proporcionar un entorno de políticas públicas propicio con acciones específicas para empoderar a las mujeres, y mejorar la gobernanza agrícola y alimentaria. Los sujetos de Parmentier no son estrictamente las comunidades de agricultores; este autor sugiere que la ampliación de la agroecología implica cambios radicales en el actual sistema agroalimentario dominante en su conjunto, incluido su sector industrial. Las diferencias entre ambos enfoques pueden basarse en el optimismo sobre la ampliación de los esfuerzos y el reconocimiento de las tensiones relacionadas con la tierra, los alimentos y el poder.

El proceso de masificación de la agroecología que se está desarrollando en Venezuela en las últimas dos décadas se caracteriza por marcos legales e institucionales promovidos por la Revolución Bolivariana (estrategia vertical) estrechamente entrelazados con un fenómeno creciente de organización social tanto en áreas rurales como urbanas (estrategia horizontal).

Cabe mencionar que la estrategia vertical se desarrolló gracias a la llegada de una nueva sabiduría política por medios democráticos en 1998; conocimiento que fue consecuencia de una crisis social, económica y política-como se menciona en Mier y Terán et al. (2018)-debida a las medidas de ajuste estructural aplicadas desde los años ochenta (Felicien et al., 2018a). Como afirma Enríquez (2013), el objetivo de esta nueva propuesta política "era mejorar las consecuencias sociales negativas del capitalismo neoliberal imperante -o poner fin al neoliberalismo en su totalidad- y reestructurar la política para incluir más a los pobres y a otros sectores marginados" (p. 612).

En este sentido, el acceso a los alimentos era una prioridad y la agroecología -definida como "agricultura sustentable" en el artículo 305 de la Constitución venezolana- se convierte en una herramienta complementaria para transformar 
gradualmente el sistema agroalimentario. Mientras tanto, los movimientos sociales están alcanzando niveles más altos de participación y deliberación, y en pocos años, diversas formas de organizaciones populares, desde Consejos Comunales locales y regionales hasta consejos de agricultores y pescadores, proporcionaron la organización social de base amplia, sugerida como un impulsor clave por Rosset (2015a), Rosset \& Altieri (2017), y Mier y Terán et al. (2018). Los discursos han desempeñado un papel fundamental en la dinamización de la agroecología en el país. Desde los discursos del presidente Hugo Chávez (19992013) que establecieron claramente una posición antiimperialista, anticapitalista y antitransgénica, y sus críticas al sistema alimentario mundial, hasta las narrativas locales de muchos agricultores, Venezuela ha dado un sólido apoyo discursivo a la agroecología. Igualmente importante ha sido el acceso masivo a la información sobre las nociones de soberanía alimentaria y sistemas agroecológicos por diversos medios. Este aspecto podría ser un factor esencial en la comprensión de la expansión agroecológica. Asimismo, se reconoce ampliamente que en América Latina los movimientos exitosos de agricultores han sido influenciados por la teología de la liberación, la cual "cuestiona fuertemente tanto el paradigma de desarrollo dominante como el camino tecnológico de la Revolución Verde" (Mier y Terán et al., 2018, p. 13).

Ambos discursos movilizadores (el del presidente Hugo Chávez y el de la teología de la liberación) comparten perspectivas no capitalistas y no imperialistas, como elementos disuasorios de la exclusión y la pobreza de los campesinos. Por lo tanto, el escalamiento de la agroecología podría considerar los discursos políticos más fuertes como un medio para lograr la expansión horizontal. Sin duda, tales discursos movilizadores amenazan los intereses corporativos e imperialistas en el sistema y los territorios agroalimentarios, provocando un poder dual, donde las clases dominantes anteriores están en conflicto con la clase emergente contendiente que aspira a conquistar el poder (Enríquez, 2013; Schiavoni, 2015).

Otro factor impulsor en el caso de Venezuela es su creencia generalizada de que el sistema dominante en el país necesita ser trasformado, lo cual es una forma continua de descolonización. La demanda de que las diversas formas de conocimiento de las comunidades indígenas, campesinas y afrovenezolanas tengan un papel más central en la vida nacional, ha impulsado gran parte del entusiasmo por la agroecología desde 1999. La dimensión de clase de la agroecología, como praxis para la supervivencia y reivindicación histórica del campesinado, la hace en especial importante para los procesos de transformación social, en los que a pesar de tener poder político, se asumen como fuerzas revolucionarias que se oponen al sistema dominante, es decir, el sistema capitalista globalizado de supremacía blanca y patriarcal. Como tal, la emergencia de un proyecto de clase amplio y contrahegemónico es un motor de primer orden de la masificación agroecológica en el contexto latinoamericano.

\section{La Universidad Bolivariana de Venezuela y el Programa de Formación de Grado en Agroecología: contexto y proceso}

La educación es una parte esencial de la estrategia para reemplazar al Estado burgués por el 
poder comunal (D'Amario, 2009). Para transformar la educación -que es un derecho-, el gobierno ha creado nuevas instituciones, facilitando su acceso, así como el desarrollo de una educación superior regionalizada (Sandoval, 2010). Los discursos de Hugo Chávez educaron al público sobre el imperativo de una transformación más profunda:

En el capitalismo, la educación es vista como una mercancía para generar ingresos en detrimento del ser humano... En el socialismo, el Estado garantiza la libertad y democratización del proceso educativo en todos los niveles, porque va más allá del valor o precio de las cosas y representa la formación integral del futuro del país (MPPRE, 2016, p. 71).

La creación de nuevas instituciones supuso una investigación crítica sobre las formas, principios y métodos de enseñanza aplicados por las universidades tradicionales. La creación de la UBV en 2003 fue un hito en la transformación de la educación universitaria venezolana, no solo por sus políticas de inclusión educativa, sino también por los ideales y prácticas de la educación universitaria. La primera tarea fue la expansión territorial de la educación superior (Chiroleu, 2009; Sandoval, 2010).

Desde esta perspectiva, el Documento Rector (2003) de la Universidad Bolivariana declaró cuatro principios para la "nueva" universidad:

(1) La educación como proceso dialógico y transformador;

(2) La educación como contextualización;

(3) La educación interdisciplinaria y transdisciplinaria, y

(4) Educación sin muros.
Estas afirmaciones condujeron al diseño de una pedagogía crítica, dialéctica y basada en el conocimiento de las personas desde su contexto social. En este sentido, se creó el PFG como una propuesta alternativa para la educación agronómica tradicional. Su objetivo principal es formar ciudadanos profesionales, con una perspectiva global que integre el conocimiento ancestral de las personas con el conocimiento moderno para mejorar los factores de producción agrícola, con respeto ecológico y comunitario. Esto permite a las generaciones futuras entablar nuevas relaciones sociales y productivas que estimulen la sostenibilidad ecológica de los sistemas agrícolas para dar respuesta a las necesidades sentidas y emergentes del proyecto del país, establecido en la Constitución de la República Bolivariana de Venezuela (UBV, 2007, p. 19).

El desafío de territorializar la formación agroecológica implicaba la elaboración de un currículo flexible que permitiera incorporar permanentemente las modificaciones regionales, según lo determinado por las realidades locales (McCune, 2016). Esto implicaba tener profesores en varias disciplinas con la capacidad de dialogar con las comunidades, para comprender e identificar las necesidades de los agroecosistemas locales desde una perspectiva holística, incluyendo las características culturales, productivas y ambientales (Domené, 2013).

Este currículo asume principios pedagógicos críticos basados en categorías epistémicas (Freire, 2006) para lograr una ciencia práctica. Así, el PFG se constituye como un espacio prometedor para lograr el desarrollo humano integral y construir proyectos comunitarios, comprometidos con las relaciones comunidad-universidad, fundamentados 
en el conocimiento, los valores, la identidad y el intercambio cultural.

El PFG utiliza el aprendizaje por proyecto como su enfoque central. Está estructurado en unidades curriculares, como módulos de diversas disciplinas asociadas a los requerimientos educativos, integradas en un marco común (Figura 1). Un proyecto se convierte en el soporte central que dirige las exigencias de formación para construir un conocimiento situado (Haraway, 1995; DomenéPainenao et al., 2020), que se desarrolle a partir de un problema colectivo elegido teniendo en cuenta el contexto social, histórico, económico, político, ecológico, cultural e ideológico (Domené, 2013).

Los proyectos involucran metodologías como la investigación acción participativa y la

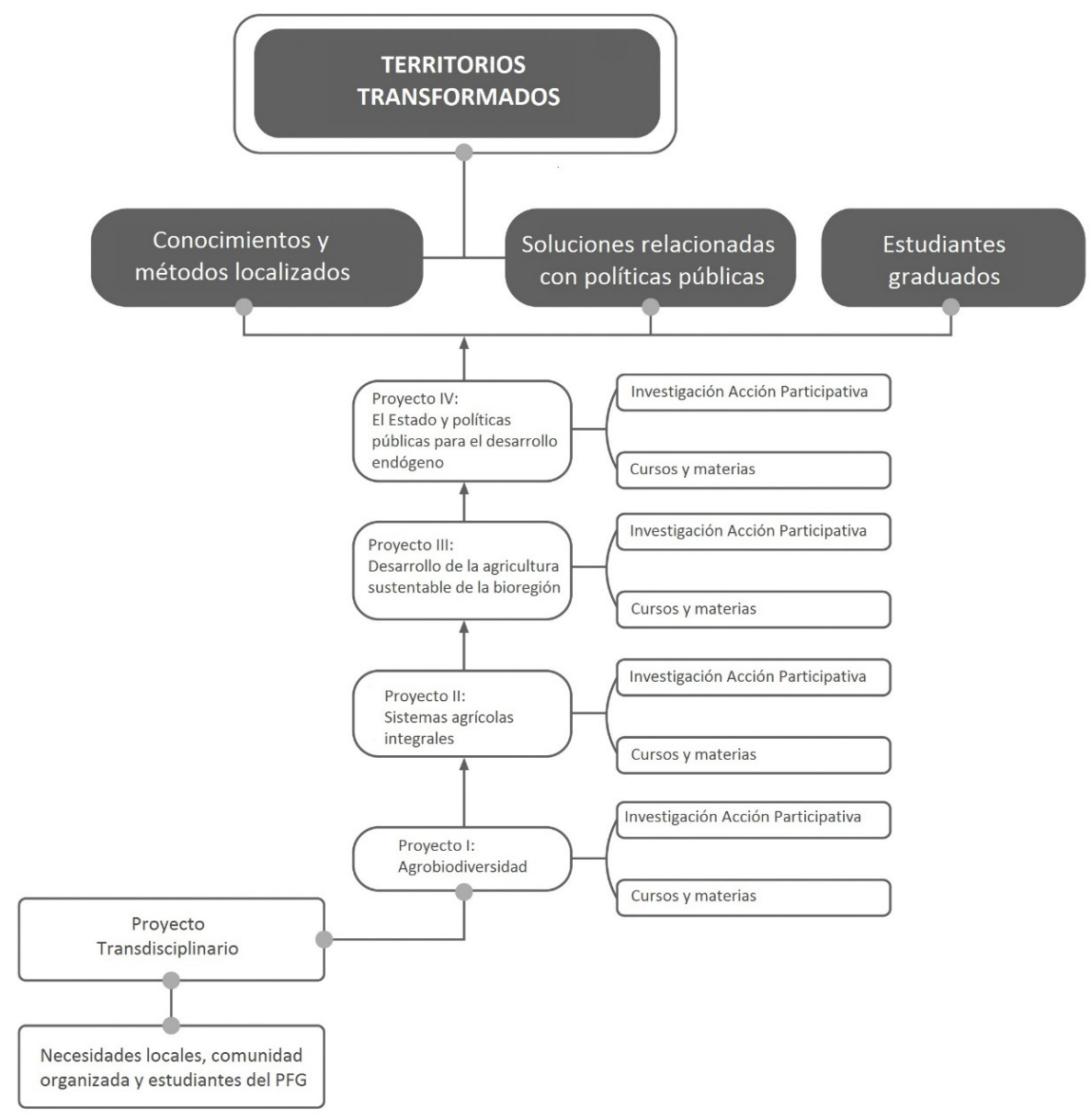

FIGURA 1 - Principales componentes del proyecto agroecológico en el Programa de Formación de Grado de la Universidad Bolivariana de Venezuela.

FUENTE: Elaboración propia. 
sistematización de experiencias, en tanto formas críticas de construir conocimiento desde la práctica (Holliday, 2012). Por lo tanto, el proyecto se basa en el método constructivista como propuesta epistemológica (Méndez et al., 2013); trata del proceso de aprendizaje individual y colectivo fundado en experiencias de vida, estructuras mentales y creencias que interpretan la realidad (Serrano \& Pons, 2011; McCune et al., 2017).

La Figura 1 muestra los componentes del aprendizaje basado en proyectos y sus relaciones con las demandas de la comunidad y las políticas públicas (Domené, 2013). En este sentido tenemos que:

- El plan de estudios está sujeto a cambios continuos y a la contextualización. Las actividades se diseñan de acuerdo con las condiciones productivas, ecológicas y ambientales de cada comunidad. Esto se hace más visible entre los ambientes rurales y los centros urbanos, donde los contenidos programáticos necesariamente cambian. Por ejemplo, los proyectos en Caracas estaban orientados a la agricultura urbana y con ellos surgieron nuevos temas como las cooperativas agrícolas urbanas, los huertos escolares y los talleres artesanales, entre otros (Alban et al., 2015).

- La continuidad del proyecto a lo largo de varios años académicos, con crecientes niveles de complejidad (UBV, 2007; Domené, 2013).

- Las políticas públicas se abordan en el cuarto año. Su inclusión en el proceso de formación brinda la oportunidad de combinar las estrategias gubernamentales en los territorios locales con las demandas de las comunidades, a través de la práctica de la agroecología. En esta fase, los alumnos estudian, construyen y proponen programas y proyectos agroecológicos para las comunas, los gobiernos municipales y otras estructuras estatales. Asimismo, con una preparación en políticas públicas se puede participar en la elaboración de nuevas leyes y otros marcos jurídicos de interés.

- Se ponen en práctica métodos participativos, con la investigación y sistematización a lo largo del proceso. En estos espacios, los diálogos incluyen aportes teóricos y la sabiduría de la comunidad para involucrarse activamente con el fin de interpretar la realidad. Un ejemplo es el caso de La Limonera en el estado de Miranda, un proyecto que se inició en 2009 y que consolidó, a través de la investigación de acción participativa agroecológica (Méndez et al., 2013), un plan de acción colectiva que culminó con la creación de una Escuela Popular de Agroecología y la gestión de una unidad productiva (Domené et al., 2009; Blanco, 2015).

- Las capacidades se desarrollan a partir de encuentros teóricos, de manera que se desenvuelven las dinámicas comunitarias vividas por los participantes. El proyecto permite un ciclo continuo de "desaprender para aprender" (McCune et al., 2014), dando una nueva lógica a los procesos. El estudiante está preparado para resolver problemas complejos, pensar críticamente, tomar decisiones e interactuar con diversas formas de conocimiento y sabiduría. Por ejemplo, un graduado ha organizado a los residentes de un gran proyecto de vivienda pública para rodear sus edificios de jardines agroecológicos (Blanco, 2015). En otro caso, un grupo de estudiantes del sur del estado de Aragua organizó un proyecto de huerta escolar municipal para el cual también producen insumos orgánicos, 
apoyando el programa de almuerzos de la escuela pública (Entrevista con la profesora Doriana Ríos, PFG Agroecología-Aragua, 2019).

- Se utilizan múltiples niveles y escalas de acción: tamaños de parcelas, asentamientos, diversidad social y biorregiones con una perspectiva compleja (UBV, 2007).

- Los proyectos se convierten en espacios de encuentro, donde se construyen diálogos y nuevos medios basados en la teoría y el conocimiento agroecológico local. La agroecología se construye como una alternativa de vida que emerge de los márgenes sociales y espacios geográficos concretos. Un profesor del campus ubicado en el estado de Zulia, al occidente del país, afirmó en una entrevista que la agroecología de las "tierras fronterizas" es muy diferente a la del centro del país. Asimismo, en el estado de Bolívar, los estudiantes cultivan alimentos en islas estacionales que se forman durante el verano en el río Orinoco, en una práctica tradicional indígena. El aprendizaje agroecológico basado en proyectos y la investigación de acción están destinados a ser capaces de adaptarse a la fenomenal diversidad de las culturas alimentarias venezolanas (Méndez et al., 2013).

Ahora bien, el aprendizaje basado en proyectos permite un proceso de "internalización y socialización de los conocimientos y la lógica agroecológicos" y coloca al territorio en el centro del proceso educativo (McCune et al., 2016, p. 17), más que al individuo, como ocurre en el sistema tradicional. En este sentido, las herramientas de aprendizaje del PFG comparten similitudes con otras experiencias latinoamericanas de formación agroecológica, como el Instituto Agroecológico Latinoamericano de la Vía Campesina
(LVC-IALA) establecida en Brasil, Paraguay, Chile y Argentina (McCune et al., 2016), y en contraste con la concepción clásica de las actividades de extensión agrícola practicadas en universidades agrícolas tradicionales (Sánchez de Mantrana, 2004).

La propuesta educativa del PFG es novedosa porque busca la construcción colectiva de las bases socioculturales y ambiental-ecológicas de una agroecología localizada y comprometida con la transformación de las relaciones sociales de producción (UBV, 2007; Domené, 2013; Herrera et al., 2017). Y eso solo es posible reconociendo a "otros" actores sociales -campesinos, mujeres rurales, pescadores, entre otros-, a través de un diálogo de saberes (UBV, 2007; Rosset, 2015a). Se trata de un desafío a largo plazo en un país petrolero rentista, particularmente dadas las tensiones geopolíticas, ideológicas, económicas y de clase que se sienten en Venezuela (Domené-Painenao et al., 2015; Schiavoni, 2015; Herrera et al., 2017). Otro tema importante en este estudio de caso es entender el proceso territorial del PFG. Uno de los principales retos de la UBV es romper la lógica de la universidad tradicional como institución hegemónica, liberal y capitalista. En el documento fundacional del PFG (UBV, 2007), esto se expresa en tres objetivos:

(1) Funcionar en todo el territorio nacional, de conformidad con lo dispuesto en la Constitución de 1999.

(2) Incorporar los territorios en un modelo de desarrollo equitativo, reduciendo la contradicción entre la ciudad y el campo y distribuyendo la inversión pública en todo el país.

(3) Priorizar los espacios locales en los procesos de interaprendizaje y establecer las condiciones 
para institucionalizar el poder popular a través de la organización de las comunas de abajo hacia arriba, tal como lo establece la Constitución venezolana (UBV, 2007).

Con estos objetivos en mente, las actividades en las zonas rurales comenzaron en 2005 en las comunidades de Barlovento, estado Miranda. Esta zona, habitada en su mayoría por afrodescendientes, se convirtió en la primera experiencia de una universidad de espacio abierto, dando sentido al lema "Universidad sin muros" del documento fundacional (Documento Rector, 2003).
Se crearon así dos escenarios distintos para el PFG: campus y ambientes. Los campus de la UBV están diseminados en la mayoría de las regiones ubicadas en casi todas las partes del país (Figura 2 ), mientras que los ambientes se ubican en los territorios rurales, cuya coordinación se asume con el compromiso de la comunidad local, así se pueden establecer en casas comunales o en parcelas rurales, de acuerdo con las demandas locales. Los ambientes son creados para satisfacer una necesidad comunitaria en la formación agroecológica y se convierten en espacios universitarios por el tiempo requerido.

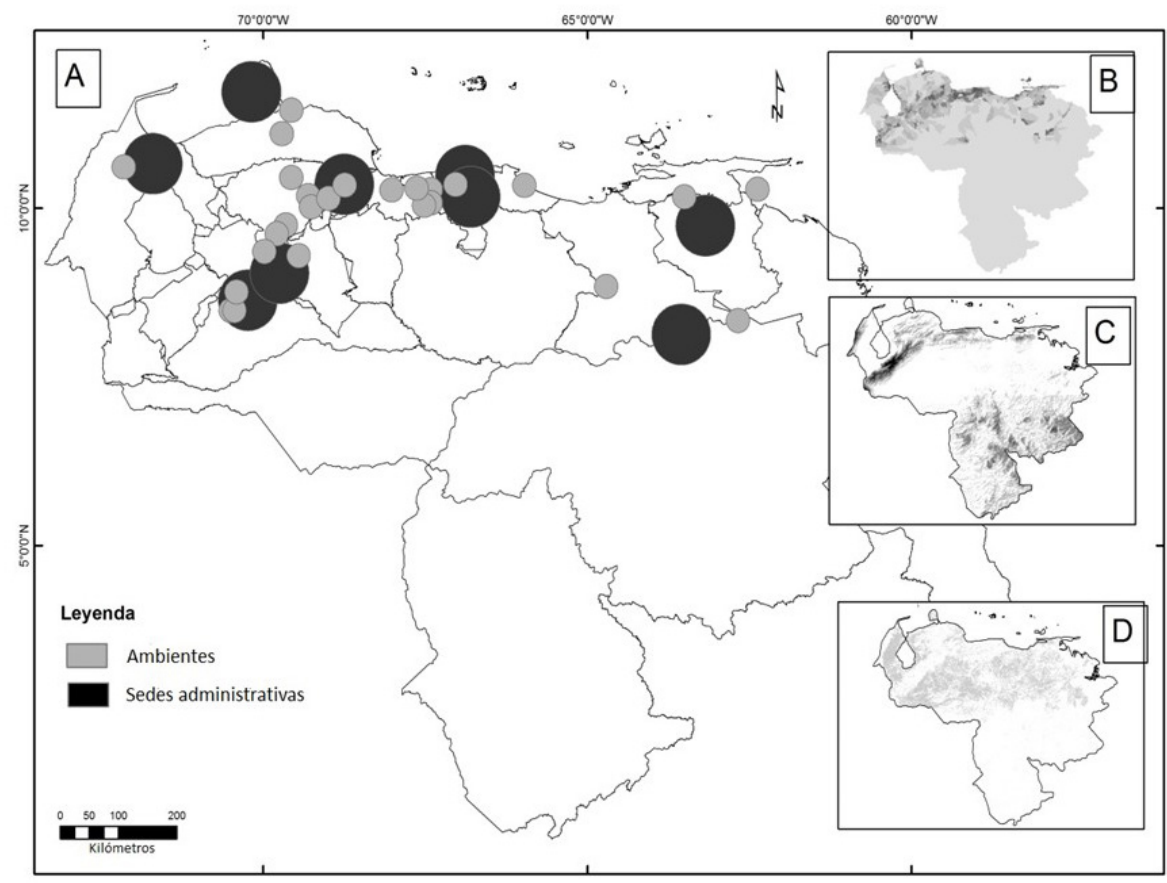

FIGURA 2 - (A) Distribución de las sedes administrativas y ambientes (escuelas Rurales) del Programa de Grado en Agroecología en Venezuela. La superficie de los círculos sugiere el área atendida por el programa (estimada en un radio de $50 \mathrm{~km}$ para las sedes administrativas y de $10 \mathrm{~km}$ para los ambientes). Los mapas anexos constituyen representaciones esquemáticas de la densidad poblacional (B), mapa físico (C) y territorios agrícolas (D) de Venezuela.

FUENTE: Elaboración propia. 
Los ambientes son cogestionados por la universidad y las comunidades. Proporcionan una enseñanza descentralizada, tanto espacial como organizativamente, en conexión con la gestión centralizada de las universidades tradicionales. En efecto, los ambientes del PFG actúan como nuevos espacios universitarios, ya que crean nuevas instituciones locales (D’Amario, 2009).

Los ambientes promueven una nueva territorialidad en los espacios rurales al utilizar el proyecto como herramienta pedagógica y el diálogo de saberes como "un método de intercambio de conocimientos, destrezas y sentidos construidos históricamente para producir colectivamente nuevos significados y luchas compartidas" (McCune et al., 2016, p. 19). Los proyectos del PFG pueden tener lugar en comunidades que se han organizado para formar comunas, una figura política creada para reflejar la necesidad de transformaciones sociales que construyan la soberanía a escalas más pequeñas (McKay et al., 2014). La cogestión comunal de ambientes ha favorecido la aparición de diversas iniciativas, como la agroecológica.

\section{Los impactos del programa de grado en agroecología}

\subsection{Población de técnicos universitarios y graduados con licencia}

Tres años después de iniciado el PFG, se graduaron los primeros 195 técnicos agroecológicos, cantidad que ascendió a 1045 después en los siguientes diez años (Figura 3). Esta cifra adquiere relevancia si se advierte que estos graduados han estado involucrados en proyectos de comunidades locales, urbanas, suburbanas y rurales a lo largo de su carrera y han tenido que lidiar con prácticas agroecológicas, políticas públicas y proyectos de investigación. Todos estos profesionales se familiarizan con la producción agrícola, las diferencias sociocomunitarias, los métodos de investigación participativa y el enfoque de diálogo de saberes entre el episteme académico y el conocimiento local.
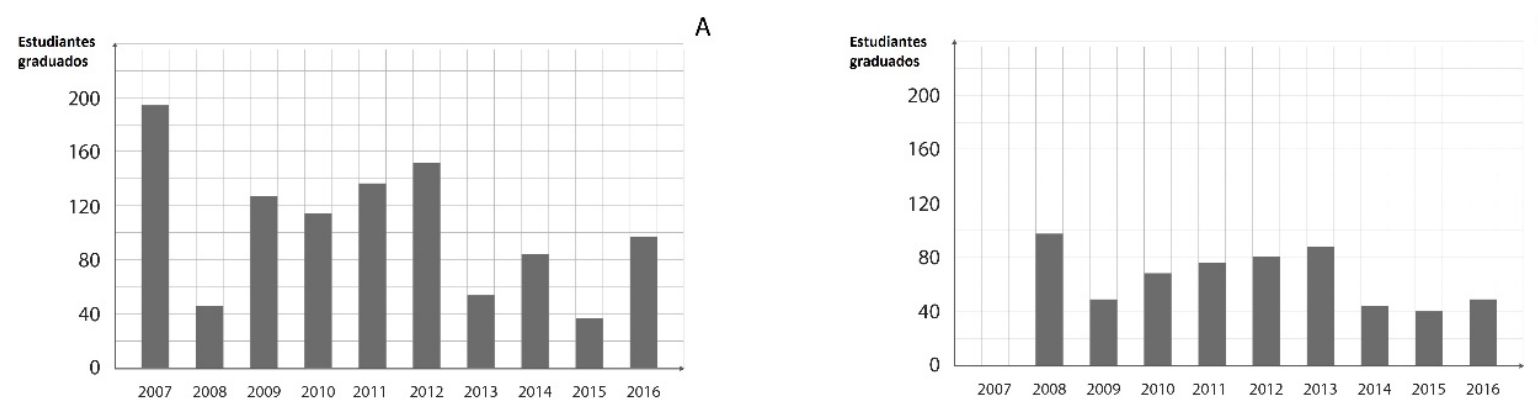

FIGURA 3 - Población de estudiantes graduados como técnicos superiores en Agroecología (A) y licenciados en Agroecología (B) en el periodo 2007-2016, en el Programa de Formación de Grado de Agroecología.

FUENTE: Elaboración própria. 
El número de graduados sugiere que la primera cohorte, como consecuencia de un proceso de acumulación, mostró un pico en el número de estudiantes. Sin embargo, este ha disminuido en los últimos años, ya que la actual crisis económica ha tomado forma. Una combinación de factores, que incluyen i) la muerte del ex presidente y líder indiscutible de la Revolución Bolivariana, Hugo Chávez, que dejó un vacío de liderazgo; ii) la repentina y dramática caída de los precios del petróleo, que dejó al Estado venezolano con menos de una cuarta parte de sus ingresos previstos en los próximos cinco años; y iii) la presión geopolítica sostenida, encabezada por los esfuerzos militares, diplomáticos, económicos, políticos y culturales de Estados Unidos para destruir la Revolución Bolivariana, se han combinado para frenar efectivamente el crecimiento del PFG.

La formación de los estudiantes ha sufrido indudablemente las consecuencias. La capacidad de respuesta del Estado para hacer frente a los acontecimientos descritos no mantuvo la producción agroecológica entre las prioridades nacionales, ni reconoció la agroecología como una política antichoque eficaz para resistir los intentos de cambio de régimen. Sin embargo, programas como Todas las Manos a la Siembra han contribuido a un vibrante movimiento de agricultura urbana basada en principios agroecológicos (Alban et al., 2017).

\subsection{Cobertura del territorio del PFG de Agroecología}

Las estrategias de inserción de la población rural y de otros sectores sociales históricamente excluidos en los estudios universitarios han sido diversas, al mismo tiempo que se tienen en cuenta objetivos específicos en los sectores rurales agrícolas, suburbanos y urbanos. La expansión del territorio ha sido un proceso gradual desde la creación del PFG en 2004, y ha sido objeto de continuos ajustes. Comenzó en cuatro ciudades: Ciudad Bolívar, Caracas, Punto Fijo y Maracaibo (Figura 2). A lo largo de los siguientes cinco años se incorporaron nuevos campus y se creó el primer ambiente. En 2011, los espacios se reorganizaron en ejes territoriales con base en criterios regionales que incluían aspectos sociales e históricos, así como la conectividad vial (García \& Santander, 2011).

La creación de ambientes rurales o urbanos es dinámica; ya que estas escuelas locales existen siempre y cuando se satisfagan sus necesidades. Así, nuevos ambientes están apareciendo continuamente en lugares donde la comunidad demanda la formación en agroecología. Las actividades del PFG han atendido a la mayoría de las áreas pobladas del país (Figura 2); especialmente en los campus principales con el apoyo de algunos ambientes urbanos; y el impacto en los sectores rurales ha sido a través de la estrategia de ambientes.

Aunque algunos ambientes han sido cerrados a resultas de la incapacidad de los estudiantes para movilizarse, también se han abierto otros (https:// cutt.ly/nb4GXAq), a menudo con un impacto más local. Donde los ambientes se han cerrado, se han creado varias iniciativas en forma de grupos y organizaciones (brigadas, escuelas abiertas, redes, etc.) que se mantienen en los espacios donde estaba presente el PFG; son emprendimientos de estudiantes que, trabajando en sus propias comunidades, aplican la agroecología como estrategia para mitigar los choques económicos que viven los venezolanos (Domené, 2016). 


\subsection{Los caminos de los ex estudiantes del PFG en Agroecología}

No hay ningún registro oficial sobre el destino de los graduados del PFG. Sin embargo, en tanto comunidad fuertemente vinculada, tomamos una muestra de los egresados a quienes entrevistamos para conocer sus opciones profesionales más comunes. Así, un profesor del PFG confirmó que casi el $90 \%$ de los estudiantes graduados de un campus urbano en el periodo 2012-2014 laboran en instituciones gubernamentales que promueven actividades agroculturales; del 10\% restante en su mayoría se ha dedicado a la producción de alimentos. Renovar las narrativas y los procedimientos de las instituciones agrícolas nacionales en áreas como la financiación, la gestión y la investigación, a raíz de las transformaciones requeridas por la Constitución, demandó un gran número de profesionales de la agroecología dentro de la gestión gubernamental. Es posible que los campus asentados en las principales ciudades del país hayan tenido una fuerte influencia en esta materia. Por el contrario, la mayoría de los graduados en los ambientes creados en comunidades rurales, aplican sus conocimientos en espacios productivos locales, en general propios. En estos casos, los graduados del PFG son campesinos con formación agroecológica, una suerte de "intelectual orgánico" para el movimiento agroecológico. Un graduado de Sanare en el estado de Lara, fundó una empresa familiar local que vende a productores de la región occidental aproximadamente 10,000 litros mensuales de fertilizantes orgánicos generados por las lombrices de tierra que cría.

\subsection{Reconfiguración de un nuevo profesional y el discurso de la transformación social}

El plan de estudios del PFG fue diseñado para desarrollar un nuevo perfil de graduados que se aleja mucho de los profesionales de la agronomía y la veterinaria egresados de los sistemas universitarios convencionales que operan bajo el paradigma de la revolución verde (Cruces, 2000). Los primeros articulan propuestas técnicas que dialogan con la sabiduría local y que emanan de diversas iniciativas comunitarias (Núñez, 2004; De Sjostrand, 2011; Rosset, 2015a) en las que el PFG se refiere como Maestro Pueblo (Núñez, 2004; Domené-Painenao et al., 2020). El reconocimiento es de otro maestro (campesinos, mujeres que curan, entre otros) que la comunidad considera sabio. La figura del Maestro Pueblo (Domené-Painenao et al., 2020) se incluyó en la experiencia formativa de la investigación acción participativa con campesinos y educadores populares en Venezuela en el contexto del PFG. Los graduados han contribuido a construir un discurso más amplio que fortalece el punto de vista agroecológico del sistema agroalimentario en su conjunto y a nivel nacional, no solo de la producción de alimentos, sino también de las nociones políticas, económicas y culturales; es un discurso que se enmarca en hechos emancipadores, antiimperialistas y soberanos.

\section{Discusión: agroecología situada en Venezuela}

Rosset \& Altieri (2017) exploran elementos de una pedagogía emergente en la formación agroecológica. Es interesante notar que la UBV, y 
en particular el PFG, han aplicado algunos de esos principios desde 2004, incrustándolos en procesos constructivistas de enseñanza-aprendizaje. En primer lugar, a pesar de un sistema universitario nacional preeminentemente conservador, la UBV adoptó el constructivismo y los enfoques freirianos como metodologías de educación alternativa. El aprendizaje basado en proyectos a lo largo de la carrera incorpora características técnico-agroecológicas, sociales y políticas basadas en escenarios agrícolas locales y globales, y perspectivas latinoamericanas -en cuanto a la comprensión de los impactos del colonialismo del conocimiento-. Este enfoque proporciona una integración holística, identidad y un sentido regional de soberanía. Aunque el PFG es una institución académica (con profesores e instructores), no puede ser considerada simplemente como una institución técnica o una extensión, ya que se ha hecho un esfuerzo notable para desmantelar esta práctica convencional del sector agrícola de la academia, en favor de una pedagogía horizontal.

Los ambientes como universidades locales, aportan la flexibilidad entre la educación formal y no formal, entre el aula y el trabajo agrícola, y crean las condiciones para intercambios horizontales de experiencias y diálogos entre diferentes conocimientos. El reconocimiento mutuo entre académicos, comunidades rurales y urbanas, estudiantes y agricultores, se beneficia de estas relaciones emergentes en ambientes del PFG.

Se han conformado ambientes PFG en comunidades rurales, suburbanas y urbanas. Por lo tanto, las dimensiones de la soberanía alimentaria y la construcción de un nuevo modelo social a través de la agroecología no se limitan a los campesinos (Rosset \& Altieri, 2017). Los agricultores y los ciudadanos pobres son fuerzas vivas para la transformación a través de su condición material y el empoderamiento de su identidad. Los profesores del PFG, además de capacitar en prácticas ecológicas, juegan un papel social y político fundamental para deconstruir las identidades de los consumidores y construir una identidad nacional sobre la base de la lucha por la soberanía alimentaria. La necesidad de soberanía alimentaria en Venezuela ha fomentado un movimiento creciente de agricultura urbana, con muchos espacios estrechamente relacionados con el PFG (Alban et al., 2017; Felicien et al., 2018a), de tal magnitud que determinó la creación de un Ministerio de Agricultura Urbana en 2015, con puntos de vista agroecológicos y conformado por un alto número de graduados del PFG.

La combinación de factores como la formación de estudiantes a través de proyectos, estrategias territoriales que combinan campus y ambientes a nivel nacional, la inclusión de los graduados en el gobierno, la dedicación de estos a la producción de alimentos e insumos orgánicos, así como encuentros y debates regionales y nacionales, sugieren que el PFG ha tenido un gran impacto en la escala horizontal de la agroindustria en todo el país. Estas actividades están concientizando a la gente sobre la crisis alimentaria y del valor de las organizaciones sociales, ayudándoles a cuestionar el modelo agroalimentario corporativo, construyendo la estructura para una masa crítica que participe en las elecciones de hoy y construyendo propuestas alternativas: conucos o jardines hortícolas, mercados hortícolas, sistemas de trueque, producción de semillas, entre otros. Como resultado, Venezuela es hoy un mosaico de experiencias alternativas relacionadas con la producción y el consumo de alimentos. 
La experiencia del PFG ha demostrado ser una estrategia eficaz para llevar la agroecología a escala desde la plataforma educativa. Desde 2004, el programa ha implementado un sistema educativo dinámico y avanzado, entrelazado con iniciativas de instituciones gubernamentales, académicos y de la sociedad civil organizada.

Insertados en esta metodología, destacan las tres ediciones del Congreso Venezolano de Agroecología (2014, 2016 y 2019), así como las dos del Simposio Nacional de Agroecología (2015 y 2018). Estos eventos han tenido diversos objetivos: en primer lugar, permiten a los estudiantes y graduados del PFG mostrar los resultados de sus proyectos de investigación, presentados con aportes de los sectores académico, institucional y campesino, y en segundo, a medida que los eventos los difunden los medios de comunicación, la agroecología gana visibilidad y se legitima. Este intercambio de experiencias subjetivas y metodológicas, de conocimientos prácticos y complejidad teórica, construyen la aceptación creativa de la sabiduría agroecológica. Se genera un tejido entre actores, dando así a la agroecología en Venezuela un carácter de movimiento social.

La inclusión de nuevos sujetos rurales ha producido una forma de "agroecología situada", en la que se entiende que el conocimiento basado en el lugar involucrado en la producción agroecológica tiene un vínculo directo con el proceso histórico y social del que surge. En otras palabras, la agroecología situada que se visibiliza durante los procesos formativos del PFG reconoce la gran diversidad de experiencias y formas de conocimiento que integran a la agroecología en Venezuela, al mismo tiempo que reconoce el compromiso con la justicia social que emana de su megadiversidad de experiencias históricas. La literatura sobre la agroecología política ha reconocido la posibilidad teórica de una agroecología situada, aunque con otros nombres (Giraldo, 2019); la experiencia venezolana ha mostrado una fuerte evidencia de la agroecología situada en la práctica (Felicien et al., 2018b; Domené-Painenao et al., 2020).

En América Latina se están acumulando evidencias de golpes o abusos llevados a cabo por fuerzas militares contra políticas o movimientos de base a favor de la agricultura alternativa particularmente en Brasil, Paraguay, Colombia y Honduras-, lo que demuestra cuán poderoso sigue siendo el modelo de la revolución verde y cuán intrincada es su red de alianzas (Giraldo \& McCune, 2019). En el contexto de la actual crisis, los venezolanos de todas las vertientes políticas tienden a sospechar que un nuevo gobierno instalado por intereses extranjeros reconstruiría los intereses de los agronegocios y reprimiría a los movimientos sociales. Esto es particularmente cierto en el caso de las organizaciones miembros de La Vía Campesina, que han hecho llamamientos muy fuertes a la resistencia popular contra lo que describen como un golpe de Estado respaldado por Estados Unidos (CLOC, 2019). El poder de los agronegocios no es local, sino global. Para desafiar al agronegocio corporativo y al sistema agroalimentario industrial, como sugieren Mier y Terán et al. (2018), la sabiduría agroecológica debe alcanzar niveles más altos de conciencia política y cultural, y necesita de sus movimientos a escala nacional y global si se quiere alcanzar el éxito. La comprensión de los factores clave y la implementación de estrategias para la ampliación de la agroecología deben estar a la altura de la tarea. A pesar de ello, los procesos de masificación en Venezuela desde 1999 han sido 
notables y muy mal documentados; existe un vacío en la literatura que este artículo intenta comenzar a llenar. La expansión de la agroecología en Venezuela no está exenta de las tensiones y presiones internas del modelo agrícola convencional, así como de los intereses nacionales e internacionales por una política de importación de alimentos que genera un doble poder (Enríquez, 2013; Felicien et al., 2018a). A esto se suma la presión de los poderes hegemónicos que exigen que la experiencia social y política de la Revolución Bolivariana sea totalmente aniquilada y desacreditada, y con ello la propuesta de la agroecología como modelo alternativo. El desafío de construir un movimiento agroecológico capaz de ser efectivo en las complejas condiciones históricas de Venezuela y bajo amenaza de invasión es altamente relevante para muchas partes del mundo que se enfrentan a los efectos simultáneos del cambio climático y la guerra económica. Quizás estos valores atípicos, los "eslabones más débiles" de las cadenas de la ecología mundial capitalista (Moore, 2015), se encuentran entre los medios más fértiles para transformaciones agroecológicas socialmente comprometidas y situadas.

\section{Agradecimientos}

Agradecemos a Nils McCune sus comentarios sobre el manuscrito. Estamos en deuda con Robert Porras y Grisel Velázquez por su ayuda con las ilustraciones.

\section{Referencias}

Alban, R.; Arteaga, M. A.; Herrera, F. F. La agricultura urbana en Caracas: diagnóstico de los espacios agroproductivos desde una perspectiva socio-ecológica. Cuadernos de Desarrollo Rural, 14(80), 1-19, 2017.

Alban, R.; Eriné, O.; Ulloa, R.; Vásquez, L. Experiencias comunales en la construcción del laboratorio artesanal de bioinsumos Catia 5, Caracas DC. En: Anales del V Congreso Latinoamericano de Agroecología-SOCLA. La Plata, 7 al 9 de oct. de 2015.

Blanco, D. Experiencia en la Producción orgánica de Hortalizas en Casas de Cultivo, en el AVIVIR La Limonera, Baruta, Miranda, Venezuela. Cadernos de Agroecologia, 9(4), 2015. Disponible en: http://revistas.abaagroecologia. org.br/index.php/cad/article/view/

Chiroleu, A. La inclusión en la educación superior como política pública: tres experiencias en América Latina. Población, 9(3.1), 17-30, 2009.
CLOC - La Coordinadora Latinoamericana de Organizaciones del Campo. La CLOC -Vía Campesina denuncia y rechaza la injerencia imperialista y exige respeto a la Soberanía de Venezuela. 2019. Disponible en: <https:// viacampesina.org/es/la-cloc-via-campesina-denuncia-yrechaza-la-injerencia-imperialista-y-exige-respeto-a-la->. Acceso: abr. 2019.

Contreras, M. A. Ciudadanía, Estado y democracia en la era neoliberal: dilemas y desafíos para la sociedad venezolana. En: Mato, D. (Ed.). Políticas de ciudadanía y sociedad civil en tiempos de globalización. Caracas, Venezuela: FACESUCV, 111-132, 2004.

Corden, W. M. Booming sector and Dutch disease economics: Survey and consolidation. Oxford Economic Papers, 36(3), 359-380, 1984. doi: 10.1093/oxfordjournals. oep. a041643 
Cruces, J. M. La enseñanza agrícola superior en Venezuela ante los desafios de la nueva agricultura. El caso de las escuelas de agronomía y producción animal. Caracas, Tesis ( $\mathrm{PhD}$ en Estudios de Desarrollo) - UCV, 2000.

D'Amario, D. Cuestiones de la inclusión educativa: A propósito de la UBV y Misión Sucre. Revista Venezolana de Economía y Ciencias Sociales, 15(1), 187-223, 2009.

De Sjostrand, M. Educación universitaria en Venezuela, 2000-2010: Logros y compromisos. Espacio abierto, 20(2), 343-365, 2011.

Documento Rector-Universidad Bolivariana de Venezuela. Documento Rector: Un proyecto educativo para la sociedad venezolana en pro del desarrollo integral del país, la trasformación del Estado venezolano y la creación de cultura democrática. Caracas, Venezuela: Ediciones UBV, 2003.

Domené, O. E. Aprendizaje por proyecto, un modelo para redescubrir la agroecología: Un avance en la evaluación de una experiencia campesina en Sabana de Uchire, Edo. Anzoátegui, Tesis (M. Sc en Desarrollo Rural) - UCV, 2013. Disponible en: http://hdl.handle.net/123456789/3704

Domené, O. Repensar el Programa de Formación en Agroecología: experiencias de proyectos hacia nuevas formas de re-construir lo alimentario en lo local. Cadernos de Agroecologia, 10(3), 2016. Disponible en: http://revistas. aba-agroecologia.org.br/index.php/cad/article/view/18985

Domené-Painenao, O.; Cruces J. M.; Herrera, F. F. La agroecología en Venezuela: Tensiones entre el rentismo petrolero y la soberanía agroalimentaria. Agroecología, 10(2), 55-62, 2015.

Domené-Painenao, O.; Mier y Terán, M.; Cacho, T. G.; Limón-Aguirre, F.; Rosset, P.; Contreras-Natera, M. Construcción territorial de agroecologías situadas: El Maestro Pueblo en Sanare, estado Lara-Venezuela. Estudios Sociales. Revista de Alimentación Contemporánea y Desarrollo Regional, 30(5-6), 2020.

Domené, O.; Riaño, A.; Pérez, O. La Limonera: una experiencia desde la organización comunitaria, para el desarrollo de una agricultura periurbana sustentable. Revista Brasileira de Agroecologia, 4(2), 4311-4314, 2009.
Enríquez, L. The paradoxes of Latin America's 'Pink Tide': Venezuela and the project of agrarian reform. Journal of Peasant Studies, 40(4), 611-638, 2013. doi: 10.1080/03066150.2012.746959

Espinoza, A. M. La cuestión agroalimentaria en Venezuela. Nueva Sociedad, 223, 128-146, 2009.

Felicien, A.; Schiavoni, C. M.; Romero, L. Food politics in a time of crisis: Corporate power vs. popular power in the shifting relations of state, society and capital in Venezuela's food system. En: Anales del International Conference Authoritarian Populism and the Rural World. Holanda, 17-18 de mar., 2018a.

Felicien, A.; Schiavoni, C.M.; Ochoa, E; Saturno, S.; Omaña, E.; Requena, A.; Camacaro, W. Exploring the 'grey areas' of state-society interaction in food sovereignty construction: The battle for Venezuela's seed law. Journal of Peasant Studies, 47(4), 1-26, 2018b. doi: $10.1080 / 03066150.2018 .1525363$

Freire, P. Pedagogía de la autonomía: saberes necesarios para la práctica educativa. México: Siglo XXI Editores, 2006.

García, S.; Santander, J. C. Los ejes geopolíticos regionales y sus ejes municipales. Caracas, Venezuela: UBV, 2011.

Giraldo, O. F. Political ecology of agriculture: agroecology and post-development. Cham: Springer, 2019. doi: 10.1007/978-3-030-11824-2

Giraldo, O. F.; McCune, N. Can the state take agroecology to scale? Public policy experiences in agroecological territorialization from Latin America. Agroecology and Sustainable Food Systems, 43(7-8), 1-25, 2019. doi: 10.1080/21683565.2019.1585402

Haraway, D. Ciencia, cyborgs y mujeres: La reinvención de la naturaleza. Madrid: Ediciones Cátedra, 1995.

Herrera, F. F.; Domené-Painenao, O.; Cruces, J. M. The history of agroecology in Venezuela: A complex and multifocal process. Agroecology and Sustainable Food Systems, 41(34), 401-415, 2017. doi: 10.1080/21683565.2017.1285842

Holliday, Ó. J. Sistematización de experiencias, investigación y evaluación: aproximaciones desde tres ángulos. $F(X)=$ Educación Global Research, 1, 56-70, 2012. 
McCune, N. Family, territory, nation: Post-neoliberal agroecological scaling in Nicaragua. Food Chain, 6(2), 92-106, 2016. doi: 10.3362/2046-1887.2016.008

McCune, N.; Reardon, J.; Rosset, P. Agroecological formation in rural social movements. Radical Teacher, 98, 31-37, 2014. doi: 10.5195/rt.2014.71

McCune, N.; Rosset, P. M.; Salazar, T. C.; Morales, H.; Saldívar, A. The long road: Rural youth, farming and agroecological formation in Central America. Mind, Culture, and Activity, 24(3), 183-198, 2017. doi: 10.1080/10749039.2017.1293690

McCune, N.; Rosset, P.; Salazar, T.; Saldívar A.; Morales, H. Mediated territoriality: Rural workers and the efforts to scale out agroecology in Nicaragua. The Journal of Peasant Studies, 44(2), 354-376, 2016. doi: 10.1080/03066150.2016.1233868

McKay, B.; Nehring, R.; Walsh-Dilley, M. The 'state' of food sovereignty in Latin America: Political projects and alternative pathways in Venezuela, Ecuador and Bolivia. The Journal of Peasant Studies, 41(6), 1175-1200, 2014. doi: 10.1080/03066150.2014.964217

Méndez, V. E.; Bacon, C. M.; Cohen, R. Agroecology as a transdisciplinary, participatory, and action-oriented approach. Agroecology and Sustainable Food Systems, 37(1), 3-18, 2013.

MPPRE - Ministerio del Poder Popular para Relaciones Exteriores. Presentación Nacional Voluntaria (PNV) ante el Foro Politico de alto nivel sobre Desarrollo Sustentable de Naciones Unidas, 2016. Disponible en: < https://cepei. org/wp-content/uploads/2019/10/Venezuela.pdf > . Acceso: ene. 2019.

Mier y Terán, M.; Giraldo, O.; Aldasoro, M.; Morales, H.; Ferguson, B.; Rosset, P.; Khadse, A.; Campos, C. Bringing agroecology to scale: Key drivers and emblematic cases. Agroecology and Sustainable Food Systems, 42(6), 637-665, 2018. doi: $10.1080 / 21683565.2018 .1443313$

Moore, J. W. Cheap food and bad climate: From surplus value to negative value in the capitalist world-ecology.
Critical Historical Studies, 2(1), 1-43, 2015. doi: 10.1086/ 681007

Núñez, J. Los saberes campesinos: implicaciones para una educación rural. Investigación y Postgrado, 19(2), 13-60, 2004.

Parmentier, S. Scaling-up agroecological approaches: What, why and how? Belgium: Oxfam Solidarity, 2014.

Rosset, P. Epistemes rurales y la formación agroecológica en la Vía Campesina. Ciência \& Tecnologia Social, 2(1), 4-1, 2015a.

Rosset, P. Social organization and process in bringing agroecology to scale. Agroecology for food security and nutrition. Rome: FAO, 2015b. Disponible en: <http://www. fao.org/3/a-i4729e.pdf>. Acceso en: Feb. 2019

Rosset, P.; Altieri, M. A. Agroecology: science and politics. Manitoba, Canada: Fernwood Publishing, 2017.

Sánchez de Mantrana, M. La extensión universitaria en Venezuela. Educere, 8(24), 83-94, 2004.

Sandoval, M. Las transformaciones de la educación superior en Venezuela: en búsqueda de su identidad. Revista Educación Superior y Sociedad, 15(1), 107-128, 2010.

Schiavoni, C. Competing sovereignties, contested processes: Insights from the Venezuelan food sovereignty experiment. Globalizations, 12, 466-480, 2015. doi: 10.1080/ 14747731.2015.1005967.

Serrano, J.; Pons, R. El Constructivismo hoy: enfoques constructivistas en educación. Revista Electrónica de Investigación Educativa, 13(1), 1-27, 2011.

UBV - Universidad Bolivariana de Venezuela. Pensum PFG Agroecología. Caracas, Venezuela: Ministerio del Poder Popular de la Educación Superior, 2007.

Verger, A.; Muhr, T. Educación Superior en Venezuela: Rompiendo con la ortodoxia liberal en política educativa. En: Merino, E. (Ed.). Venezuela como laboratorio de políticas públicas: seis miradas a la sociedad, la economía y la educación bolivariana, Málaga, España: Centro de Ediciones de la Diputación de Málaga, 67-103, 2007. 